\title{
SENTINEL-1 BASED FLOOD MAPPING USING INTERFEROMETRIC COHERENCE AND INTENSITY CHANGE DETECTION APPROACH
}

\author{
I. Papila ${ }^{1, *}$, U. Alganci $^{1}$, E. Sertel ${ }^{1}$ \\ ${ }^{1}$ ITU, Research and Application Center for Satellite Communications and Remote Sensing (CSCRS), 80626 Maslak Istanbul, \\ Turkey - (papila, ugur, elif)@ cscrs.itu.edu.tr
}

KEY WORDS: Flood Mapping, Interferometric Coherence, SAR Intensity, Region Growing Algorithm, Change Detection, Sentinel-1, Spot-6

\begin{abstract}
:
This study presents a semi-automatic algorithm for mapping floods. Both Optical and Synthetic Aperture Radar (SAR) data are used to observe the flood that hit the Cukurova region of Adana (Turkey) in 2019. The performance of the interferometric coherence in complementing intensity component of SAR data is investigated for mapping the floods occurred in agricultural and urban environments. There was no ground truth data available from the flooded area, thus classification result of optical satellite image is used as a seed for the region growing algorithm that defines the classes according to a threshold value. The advantage of using both intensity and coherence change detection is verified with the results. The results have been evaluated through very high-resolution SPOT-6 optical image which acquired simultaneously with Sentinel-1B SAR image. The comparison with the SPOT-6 data results shows that the proposed approach can map flooded areas with acceptable accuracy using the SAR data from Sentinel-1 satellite mission. Highly affected agricultural areas along with the river line could be mapped both by optical and SAR analysis. Comparison of results from VV and $\mathrm{VH}$ polarization provided that cross-polarization $\mathrm{VH}$ has a very little effect on flood mapping. The proposed algorithm successfully distinguishes the classes among the affected region, especially in urban areas.
\end{abstract}

\section{INTRODUCTION}

\subsection{General Instructions}

Synthetic Aperture Radar (SAR) and optical satellite data have proven to provide essential information in case of natural disasters like flooding. Recently floods are occurring more often due to changes in climate and land-use characteristics. Such disasters can have a strong impact on crops, housings and even public health. Flood monitoring and mapping can help organizations and authorities in disaster management. Moreover, highly accurate flood mapping provides key information in strategic planning for the authorities for damage estimation and sustainable urban planning to properly manage flood risk. Satellite images enable fast, reliable and costeffective monitoring of large coverages, thus provide geospatial information to support disaster monitoring and management in an effectively (Amadio, 2016). Identification of flooded areas become much effective and profitable by use of multispectral satellite data, Synthetic Aperture Radar (SAR) data or a combination of them (Notti et al., 2018), (Rahman and Li, 2017), (J Li et al., 2018,) and (Muro et al., 2016). Many studies have proven that SAR systems are very effective and reliable tools for flood mapping especially on vegetated areas and the bare soil as well, by benefiting from their sensitivity to surface roughness and soil moisture changes (Schlaffer et al., 2015) and (Li et al., 2018).

Instead of using a single image, change detection-based methods have an advantage in masking out false alarms like permanent water areas and look-alikes. Especially on bare soils, one SAR observation can be enough for flood mapping. However, in urban and agricultural areas, at least one pre-flood image and one after-flood image would provide more reliable results in a sense of change detection approach. Change detection based on multi-temporal SAR images is widely used for disaster monitoring. SAR-based change detection methods require repeated acquisitions that provide a static background against the surface change that can be distinguished by using the change indicator. Two types of change detection approaches can be performed by use of SAR data. First is the incoherent (amplitude) change detection (ACD), which compares the backscatter between two scenes and find out the changes over the region. The capability to detect the changes is very limited with this method. Second is the coherent change detection (CCD), which computes the phase coherence between the two scenes. Since the phase is very sensitive to minor changes, any decorrelation in the phase will result in coherence change. Among the InSAR techniques, coherence is one of the important components that is used to discriminate flooded and non-flooded areas (Olen and Bookhagen, 2018). Changes of the Earth's surface will cause a loss of coherence between two images that are collected before and after the hazard.

In sense of feature selection to define and model the change between two scenes; there are several methods used in literature both for pixel-based or texture-based models (Ouled Sghaier et al., 2018) and (J Li et al., 2018). Commonly used change indicators for pixel-based models are the ratio, log-ratio $(\mathrm{Y} \mathrm{Li}$, 2018) or difference operator, which suppresses the background information to extract the changed region. The histogram-based thresholding and the distance-based clustering are also frequently used methods to extract the change information. The region growing is proposed to improve the pixel-based solutions. The drawback of the pixel-based method is the performance fails due to the speckle noise. On the other hand, texture-based descriptors are difficult to implement due to their complexity. Commonly used texture-based descriptors (Ajadi et al., 2016) are mathematical morphology operators, second-order 
texture parameters, statistical and signal processing models; like co-occurrence matrix, wavelets, independent component analysis and Gabor feature. Conditional Random Field (CRF) (Y Li, 2018) is another contextual based model that is applied for both SAR image classification and detection. A statistical model like Expectation-Maximization (EM) is also applied with Markov Random Field to detect the changes in SAR images. Fuzzy logic classifier is also used in unsupervised change detection of SAR images (Twele et al., 2016).

Coherence studies monitoring the surface damage have typically use the longer-wavelength (L-band) SAR satellites such as ALOS. The shorter wavelength X-band systems such as TerraSAR-X or COSMO-SkyMed are sensitive for urban and vegetation-free areas (Chaabani et al, 2018). On the other hand, C-band radar systems (Sentinel-1) (Borah et al., 2018) and (Monti-Guarnieri et al., 2018) have a higher sensitivity to surface changes and vegetation cover, which can produce a noise signal and cause potential challenges for observation. Despite these disadvantages, Sentinel-1 data are freely available and have a higher repeat time (6-12 days) [3]. In addition to sensor parameters, the measurement of backscattering parameter depends on imaging geometry, surface roughness, and the polarization (Kornelsen and Coulibaly, 2013). Although horizontal transmit-horizontal receive $(\mathrm{HH})$ polarization is generally preferable for flood mapping (Henry et al., 2003), vertical transmit-vertical receive VV polarized data (Matgen et al., 2011) and (Schumann et al., 2009) and multi-polarized SAR data (Sree et al., 2006) and (Clement et al., 2017) have been used successfully for flood mapping in previous studies.

Several parameters affect the performance of change detection algorithms due to the nature of SAR data:

- Complicated nature of surface backscattering response

- Multiplicative speckle noise

- Hard to develop an automatic or adaptive technique to define the change detection threshold

- Lack of location accuracy to extract the boundary of changed regions

The interferometric coherence $(\gamma)$ was demonstrated recently by several researches to detect floodwater (Chini et al., 2012), (Chini et al., 2019), (Refice et al., 2014) and (Pulvirenti et al., 2016). When using the CCD approach to monitor the flooding, one important requirement for ensuring the changes in the observed region is caused by nothing but the flooding itself.

In this study, Sentinel-1B InSAR pairs were used to calculate the interferometric coherence and intensity to detect the water body for a selected threshold. To produce a homogeneous flood mapping for the affected region, a standard region-growing algorithm (RGA) is used. The RGA searches for pixels within the whole SAR image that are connected neighbours to the pixels belonging to a seed region and that fall within a tolerance criterion (Haralick 1985). In addition, SPOT-6 optical data was used for water body detection, later used for seeding and the accuracy analysis of the proposed method.

\section{STUDY AREA AND DATASET}

\subsection{Study Area}

We are interested in the region of Adana, Mediterranean city from the Southern part of Turkey. It is part of a historical region called Çukurova Plain. With its vast, fertile lands and important rivers such as Seyhan, Ceyhan and Göksu, the Çukurova Plain is one of the most productive areas of the world in terms of agriculture. Almost every agricultural product of Turkey such as cotton, soy, peanuts, corn, wheat, maize and various fruits and vegetables could be produced in Çukurova. The area suffered from temporally flooding during January 2019 due to high amount of precipitation (Figure 1). The SAR data used in this study concern the overflowing of the Seyhan River, due to the widespread and persistent rainfall, which occurred January 1516, 2019.

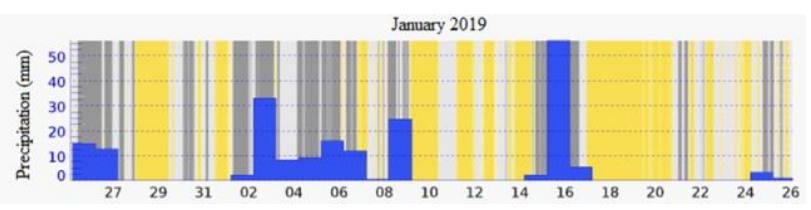

Figure 1. Adana monthly precipitation in January 2019

\subsection{Dataset}

The proposed methods were tested and evaluated on Sentinel-1 C-band dual-polarization Interferometric Wide (IW) swath mode Single Look Complex (SLC) datasets over the Seyhan River, Turkey. Radiometric calibration, speckle filtering, binarization, co-registration, Range-doppler terrain correction were performed within the Sentinel Application Platform (SNAP). During the pre-processing step, the Boxcar filter with a $5 \times 5$-window size was used to remove the speckle noise.

The 20 January 2019 dated SPOT 6 image of the study region was acquired as pansharpened product. This product includes blue, green, red and NIR spectral bands at $1.5 \mathrm{~m}$ spatial resolution. The first step of the pre-processing is the atmospheric correction of the satellite image in order to obtain the ground reflectance values from the quantized digital numbers. This process was performed using the ATCOR module of PCI Geomatica software. In the second step of the pre-processing, the image was orthorectified using the rational function (RF) model constructed from RPC coefficients and ALOS - W3D DEM data.

Properties of the data that were used in this study shown in Table 1.

\begin{tabular}{|l|l|l|l|l|}
\hline Satellite & Product & Date & Polarization & Data ID \\
\hline S-1B & SLC-IW & 26.12 .2018 & VV+VH & S1 pre \\
\hline S-1B & SLC-IW & 07.01 .2019 & VV+VH & S2 pre \\
\hline S-1B & SLC-IW & 19.01 .2019 & VV+VH & S3 post \\
\hline S-1B & SLC-IW & 31.01 .2019 & VV+VH & S4 post \\
\hline SPOT 6 & PSP & 20.01 .2019 & & \\
\hline \multicolumn{7}{|l}{}
\end{tabular}

\section{METHOD}

The method based on extracting the coherence map from the consecutive interferometric pair of Sentinel-1B images. Figure 2 shows the overall process and summarizes the algorithm steps.

The backscattering coefficient and interferometry coherence estimation among the Sentinel pairs are calculated in SNAP. The centroid of each segmentation is calculated from shapefiles containing water body detection results. Then these centroid locations are used as seed for the Region Growing Algorithm that identifies the classes. 


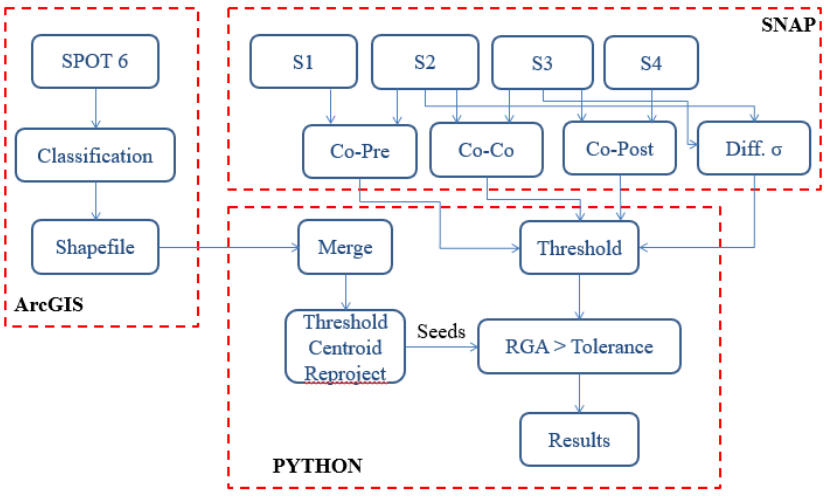

Figure 2. Block diagram of the floodwater-mapping algorithm

\subsection{Change Detection Using the Interferometric Coherence and Intensity}

Interferometric coherence measures the degree of correlation between two complex images. Interferometric pairs can be chosen before the flood occurrence (Pre-event - S1 \& S2), after the flood occurrence (Post-event - S2 \& S3) or one image before the flood and the other is after the flood (Co-event - S2 \& S3). Post-event coherence can help in distinguishing vegetated areas from bare surfaces in agricultural areas. The algorithm is based on the detection of pixels belonging to the following backscatter $\left(\sigma^{\circ}\right) /$ coherence $(\gamma)$ classes for agricultural and urban areas. These classes were identified and introduced by Pulvirenti et al (2016).

C1: $\gamma($ co-event $)<$ Threshold

C2: $\gamma$ (co-event) $-\gamma($ pre-event $)<$ Threshold

C3a: $\gamma$ (post-event) $-\gamma($ pre-event $)>$ Threshold $\quad \& \&$

C3b: $\gamma$ (post-event $)-\gamma($ co-event $)>$ Threshold

C4: $\sigma^{\circ}\left(\right.$ SAR-3) $-\sigma^{\circ}($ SAR-2) $<$ Threshold

C5: $\sigma^{\circ}\left(\right.$ SAR-3) $-\sigma^{\circ}($ SAR-2) $>$ Threshold

C6: $\gamma$ (pre-event $)<$ Threshold

C1 corresponds to the interferometric coherence between the two SAR images one of which is just before the flood and the other is just after the flood (Co-event). If there is a correlation between these two images, the coherence value will be close to 1 ; if the value close to zero this means there is no coherence between those two passes mostly due to the flood. Therefore, we aim to detect the region where the coherence value is below the threshold. (Agricultural areas and bare soils)

C2 corresponds to the interferometric coherence difference between Co-event pairs and the Pre-event pairs. The decrease in coherence can indicate a change in the scenario caused by the flood. (Urban areas)

C3 corresponds to the interferometric coherence difference between Post-event and Pre-event, and between Post-event and Co-event at the same time. Double bouncing enhancements from the flooded vegetation areas will cause high coherence at the Post-event pair, which can help to detect flooded agricultural areas with persistent standing water. (Agricultural areas)

C4 corresponds to the difference in backscattering values between after the flood image and before the flood image. The low value of the backscattering can help to detect the presence of floodwater in agricultural areas. (Agricultural areas and bare soils)

C5 corresponds to the difference in backscattering values between after the flood image and before the flood image. The presence of floodwater can cause double-bounce backscatter, which will increase the radar cross-section in an ideal situation of an isolated building surrounded by a homogenous ground surface. (Urban Areas)

C6 corresponds to the interferometric coherence between the two SAR images before the flooding (Pre-event). Coherence in the vegetated areas expected to be low when the area is not affected by the flood. The presence of the floodwater at the vegetated areas can cause the double bounce effect, which will increase the radar-cross section. (Vegetated areas)

\begin{tabular}{|c|c|c|c|c|c|}
\hline Class & Definition & Pol. & Thresh. & Tolerance & Oper. \\
\hline C1 & $\gamma(\mathrm{S} 2 \& \mathrm{~S} 3)$ & VV & 0.15 & 0.25 & $<$ \\
\hline C1 & $\gamma(\mathrm{S} 2 \& \mathrm{~S} 3)$ & $\mathrm{VH}$ & 0.15 & 0.22 & $<$ \\
\hline $\mathrm{C2}$ & $\gamma(\mathrm{Co}-\mathrm{Pre})$ & $\mathrm{VV}$ & -0.6 & -0.4 & $<$ \\
\hline $\mathrm{C2}$ & $\gamma$ (Co-Pre) & $\mathrm{VH}$ & -0.6 & -0.4 & $<$ \\
\hline C3a & $\gamma$ (Post-Pre) & $\mathrm{VV}$ & 0.65 & 0.5 & $>$ \\
\hline C3a & $\gamma$ (Post-Pre) & $\mathrm{VH}$ & 0.65 & 0.5 & $>$ \\
\hline C3b & $\gamma$ (Post-Co) & VV & 0.65 & 0.5 & $>$ \\
\hline C3b & $\gamma$ (Post-Co) & $\mathrm{VH}$ & 0.65 & 0.5 & $>$ \\
\hline $\mathrm{C4}$ & $\sigma(\mathrm{S} 3-\mathrm{S} 2)$ & VV & $-8 \mathrm{~dB}$ & $-4 \mathrm{~dB}$ & $<$ \\
\hline $\mathrm{C4}$ & $\sigma(\mathrm{S} 3-\mathrm{S} 2)$ & $\mathrm{VH}$ & $-8 \mathrm{~dB}$ & $-4 \mathrm{~dB}$ & $<$ \\
\hline C5 & $\sigma(\mathrm{S} 3-\mathrm{S} 2)$ & $\mathrm{VV}$ & $8.5 \mathrm{~dB}$ & $6.5 \mathrm{~dB}$ & $>$ \\
\hline $\mathrm{C5}$ & $\sigma(\mathrm{S} 3-\mathrm{S} 2)$ & $\mathrm{VH}$ & $8.5 \mathrm{~dB}$ & $6.5 \mathrm{~dB}$ & $>$ \\
\hline C6 & $\gamma(\mathrm{S} 1 \& \mathrm{~S} 2)$ & VV & 0.15 & 0.23 & $<$ \\
\hline C6 & $\gamma(\mathrm{S} 1 \& \mathrm{~S} 2)$ & VH & 0.15 & 0.23 & $<$ \\
\hline
\end{tabular}

Table 2. Class Thresholds and Tolerances for each polarization

The identification of classes $\mathrm{Ci}(\mathrm{i}=1: 6)$ has been carried out by applying a region growing algorithm (RGA).

Floodwater mapping results from SPOT-6 images are used to define the thresholds for each class and this information was used to determine the seed region for classes on the SAR data. Then RGA looks for pixels that are connected as neighbours to the seed regions according to a tolerance criterion.

- For agricultural zones: the area labelled as flooded if the pixels belonging to

F1: [C1 \& C4] || [C1 \& C5 \& C6] \| C3

- The area labelled flooded bare soils or plants completely submerged by water if the pixels belonging to

F2: $\mathrm{C} 1 \& \mathrm{C} 4$

- The area labelled flooded vegetated areas if the pixels belonging to

F3: $\mathrm{C} 1 \& \mathrm{C} 5$ \& $\mathrm{C} 6$

- For urban settlements the classification scheme labels as flooded if the pixels belonging to F4: C2 || $\mathrm{C} 5$

- Total area effected from flooding can be expressed as F (Total): F1 \|F2 \|F3 || F4

\begin{tabular}{|c|c|c|c|c|}
\hline & C1 \& C4 & C1 \& C5 \& C6 & C3a \& C3b & C2 $\|$ C5 \\
\hline F1 & x & X & X & - \\
\hline F2 & x & - & - & - \\
\hline F3 & - & x & - & - \\
\hline F4 & - & - & - & X \\
\hline
\end{tabular}

Table 3. Class Distribution over the land cover map 


\section{MODEL IMPLEMENTATION AND PERFORMANCE ANALYSIS}

\subsection{Classification of the flooded areas}

The Figure 3 presents the effected urban areas from the flood. The model successfully distinguishes the land cover type of the flooded region inside the urban area and assigns the class only as urban. However, in few regions, some of the flooded areas can be assigned to two or more classes, likewise in the bare soil case. If the region completely submerged by water, it would be difficult to distinguish bare lands from the agricultural area, which is affected by the flood. Figure 4 shows the affected agricultural regions, while Figure 5 shows the same area as bare soil/plant completely submerged by water

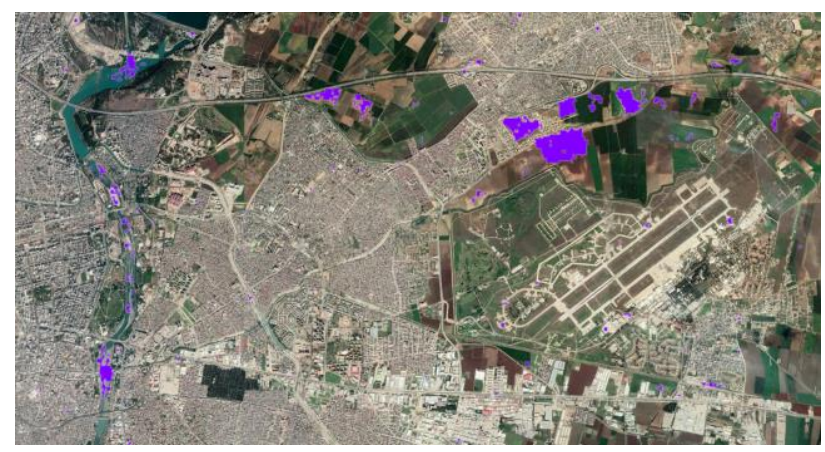

Figure 3. The urban area effected from the Flood

\begin{tabular}{|l|l|c|l|}
\hline & Legend & Polarization & \multicolumn{1}{|c|}{ Area Type } \\
\hline F1 & & Both & Agricultural \\
\hline F2 & & Both & Bare Soil / Plants \\
\hline F3 & & Both & Vegetated \\
\hline F4 & & Both & Urban \\
\hline
\end{tabular}

Table 4. Flooded Area Class Legend

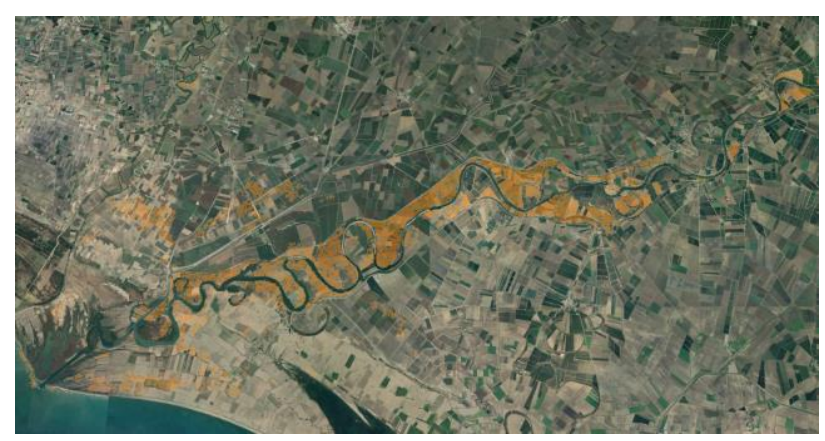

Figure 4. Agriculture zone affected by the flood

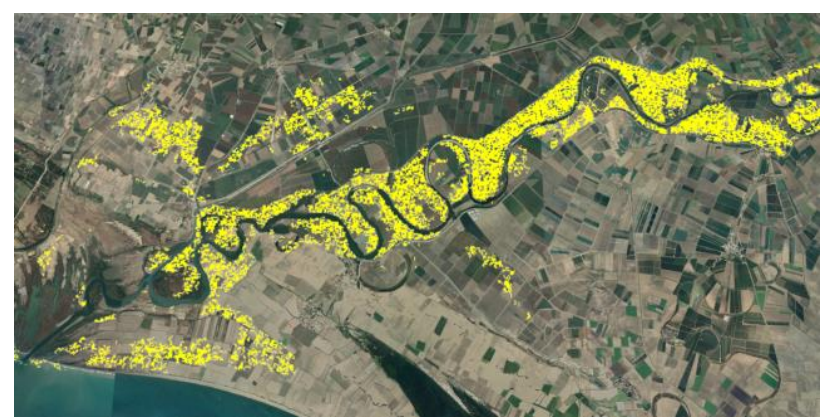

Figure 5. Bare soil zone affected by the flood

\subsection{Cross Polarization on flood mapping}

Figure 6 shows that, using both the cross-polarization and the VV polarization at the same time did not improve the flood mapping result. Moreover, VH polarization results were not successful in determining the flooded area. On the contrary, using the $\mathrm{HH}$ polarization would bring extra information about the flooded area, which would improve the algorithm performance. For the time interval, $\mathrm{HH}$ polarization was not available for the study area, so we did not have a chance to test the impact of $\mathrm{HH}$ polarization data on the proposed algorithm.

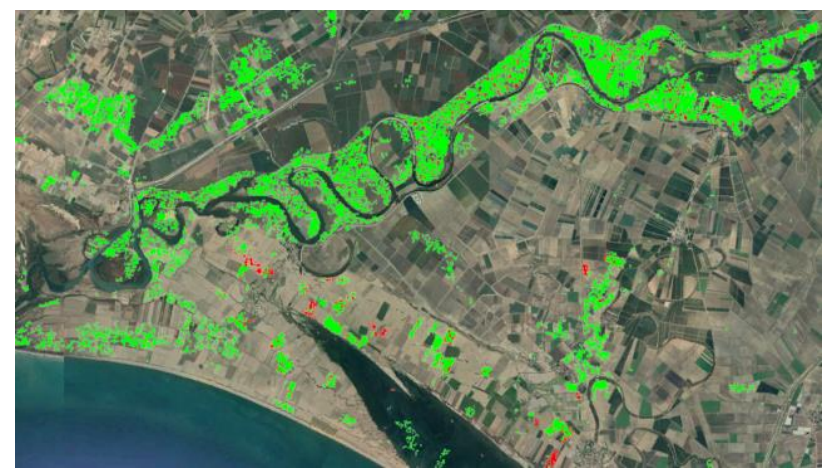

Figure 6. Cross Polarization effect over flooded area

\begin{tabular}{|c|c|c|c|}
\hline & Legend & Polarization & Area Type \\
\hline F(Total) & & VV & All \\
\hline F(Total) & & VH & All \\
\hline F(Total) & & Both & All \\
\hline
\end{tabular}

Table 5. Flooded area Polarization Legend

\subsection{Flood Mapping via SPOT 6 Satellite Image}

In flood mapping and damage assessment with use of optical satellite images, most researches focused on the classification of image bands or threshold-based analysis of spectral indices derivate of the image bands. The well-known spectral index for delineating open waters and flooded lands is introduced by Gao (1996), named normalized difference water index (NDWI). This index is based on the normalized difference of NIR and SWIR bands, thus applicable to images from Landsat and Sentinel 2 missions. Modified versions of this index were applied successfully in recent researches, where modification is applied as replacing the NIR band with green, red or red edge bands (Xu, 2006, Zhang 2016, Notti et al 2018).

McFeeters (1996) proposed different version of NDWI, which uses green and NIR image bands. This combination is applicable on high and very high spatial resolution satellite images such as SPOT, Pleiades and Worldview 4 operational missions, which do not include SWIR spectral bands (Eq. 1).

$$
\mathrm{NDWI}_{\text {McFeeters }}=(\text { Green }-\mathrm{NIR}) /(\text { Green }+ \text { NIR })
$$

The application of this index provides a value range between -1 and 1 , where positive values correspond to high moisture lands and open waters, while negative values corresponds to vegetation and dry lands. Xu (2006) reported that, although

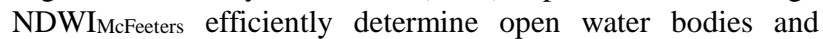
heavily flooded lands, it could not completely suppress the lower magnitude positive responses from built-up regions, which results with false positive detection of some built -up regions as water bodies. McFeeters (2013), proposed a 
binarization procedure with single threshold value of 0.3 to maximize the difference between water and non-water regions to overcome the problem. However, this approach has the risk of omitting comparatively low-level flooded areas and water bodies with high sediment concentration, which may represent lower magnitude index values $(0.10-0.30)$.

To overcome the problem of false detection of built-up regions, a modification to NDWIMcFeeters is proposed by subtraction of the normalized difference vegetation index (NDVI) that is presented in Eq. 2.

$$
\text { MNDWI }_{\text {McFeeters }}=\text { NDWI }_{\text {McFeeters }}-\text { NDVI }
$$

The NDVI produces opposite responses for the land cover classes and flooded areas compared to MNDWIMcFeeters, thus subtraction-based modification exaggerates the index intervals for surface types.

After index production, the data was classified using modified natural breaks algorithm. The natural breaks algorithm aims to cluster the data by grouping similar values while maximizing the class differences by determining the most optimal and natural class ranges (Jenks, 1967; Slocum, 2009). This phenomenon is satisfied by minimizing the within-class deviation while maximizing the deviation between class means (McMaster 1997).

In this research, initially three intervals were detected by use of natural breaks algorithm to differentiate the vegetation, bare lands, and urban areas and water bodies and flooded areas. In the second step, the initial three intervals are divided into subintervals with equal ranges, for grading purposes. A final classification legend with 16 intervals for representation is given in Figure 7.

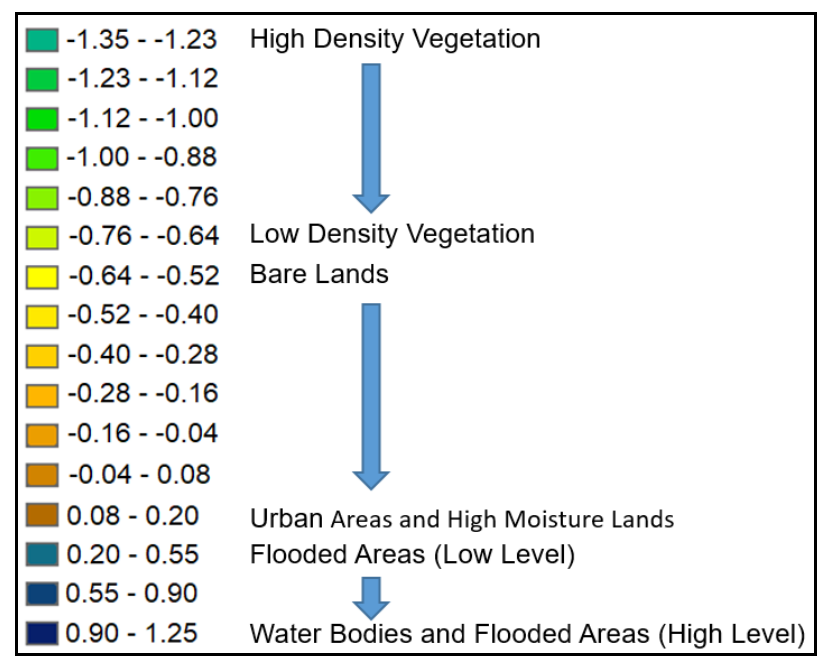

Figure 7. The classification legend for flood mapping with MNDWI $_{\text {McFeeters data. }}$

The resulting map of the study region with the false color image is given in Figure 8. The visual inspection on the map along with the original image pointed out an effective detection of the flooded areas on the land surface, well discrimination of vegetated areas and bare lands, and built up areas.

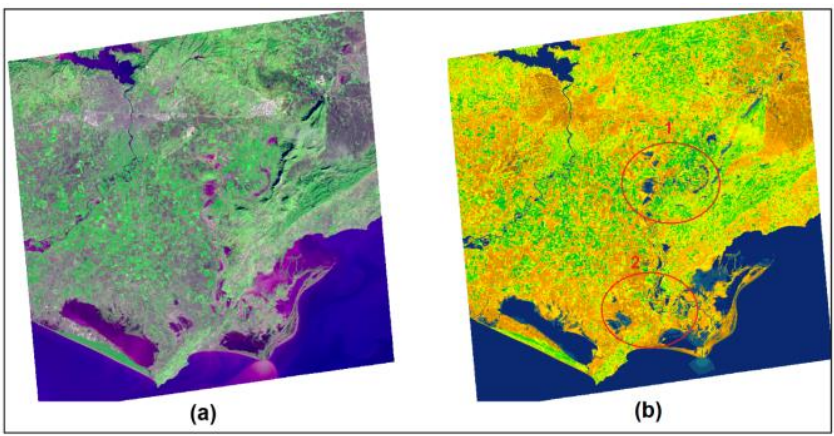

Figure 8. Overview of the study area, (a) SPOT 6 Red/NIR/Blue composite, (b) classified MNDWI McFeeters data.

Although the produced map provides good separation between above-mentioned classes, there is a need for determining the flooded land surfaces and isolate them from the open water bodies. For that purpose, the last three classes belonging to flooded areas and water bodies were extracted as a vector file, and an area-based removal analysis was performed to remove the large-sized water bodies. The extracted vector map and the result of area-based removal analysis are given in Figure 9.

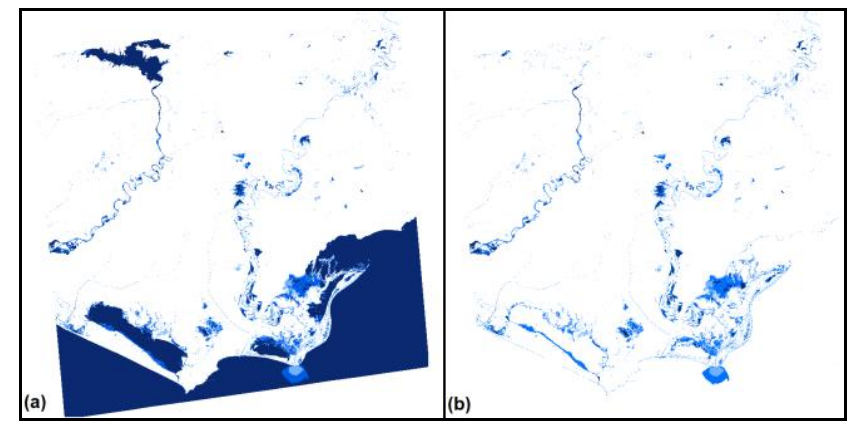

Figure 9. Target masking results, (a) water bodies and flooded areas, (b) flooded areas and rivers after open water removal.

\subsection{Accuracy Analysis: SPOT-6 vs Proposed Model}

According to the results obtained from SPOT-6 data, the number of pixels affected by the flood is 114262 and the total area affected by the flood is $108.913 \mathrm{~km}^{2}$. On the other hand, according the results obtained with the proposed method, number of pixels affected by the flood is 112633 while the total area affected by the flood is $107.278 \mathrm{~km}^{2}$. Overlapped pixels that are identified as a flooded area in both SPOT-6 and SAR results are 16849 while the overlapped area size is $16.051 \mathrm{~km}^{2}$.

However, to keep in mind the water bodies like rivers, lakes are excluded from the classification result in the proposed SAR method while it is seen from Figure 10 that the river is already included in the optical result (Blue Class). In addition, the SPOT-6 and the Sentinel coverage area do not perfectly match with each other. As can be seen on the left side of Figure 11 SPOT-6 data coverage ends on the white and green classes' border. Since the Sentinel $1 \mathrm{~B}$, coverage is bigger than the SPOT-6 in this study; it is expected to have larger affected flooded areas from the SAR analysis, which explain the green class on the left side of Figure 11.

The model performs well among the riverside. As it is seen from Figure 11, both SPOT-6 analysis results and the proposed 
method determine the flooded regions nearby the river successfully. If we look at the performance of the method in agricultural areas, we can still say that there is a correlation between the results from SPOT-6 image and the proposed method (Figure 12).

Nevertheless, more agricultural areas are determined as affected in the proposed method. This result can be also explained by uncertainties in selecting optimum values for the thresholds and tolerances in the algorithm. Moreover, there were no ground truth data available in our study, thus we relied on the results from SPOT-6 optical data classification and aimed to maximize the match between the two results. Thus, our thresholds and tolerances might be higher than the optimum values and this can lead to a false alarm on the proposed algorithm.

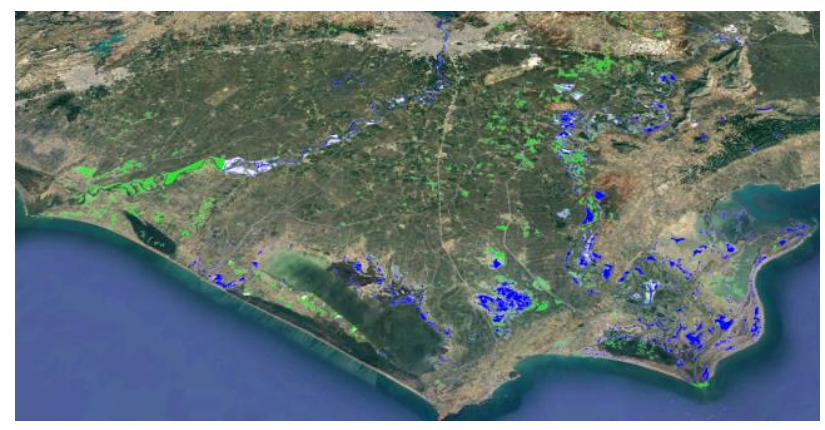

Figure 10. SPOT-6 vs SAR result

\begin{tabular}{|l|l|}
\hline Radar & \\
\hline Spot & \\
\hline Both & \\
\hline
\end{tabular}

Table 6. Spot and SAR Flooded Area Legend

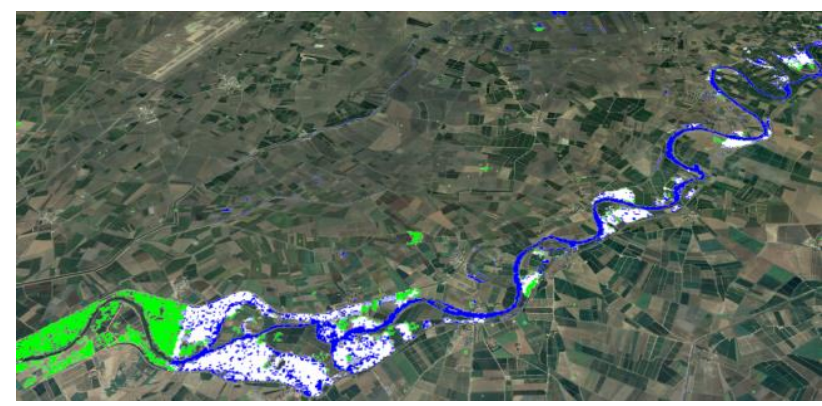

Figure 11. SPOT vs SAR Riverside Detailed

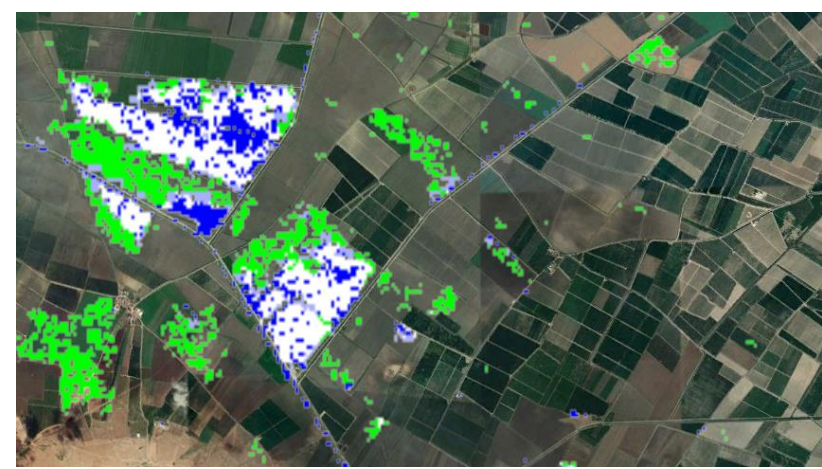

Figure 12. SPOT vs SAR Agriculture Area Detailed
Other factors that can affect the algorithm performance is choosing the optimum pairs among the SAR data. In an ideal case, both pre-event SAR images (S1 and S2) should be acquired in dry weather conditions, on the other hand, both post-event SAR images (S3 and S4) should be acquired in flood conditions. At the same time, pre-event and post-event data time intervals should not be long enough to cause an extra loss of coherence between the pairs, which makes it difficult to specify the reason for the degradation of the coherence. The more we are close to the ideal conditions; the more the coherence results would be reliable. In our case, S4 is acquired 12 days later than S3. So, the post-event coherence that is used in C3 isn't that ideal.

\section{REFERENCES}

Ajadi, O.A.; Meyer, F.J.; Webley, P.W. Change Detection in Synthetic Aperture Radar Images Using a Multiscale-Driven Approach. Remote Sens. 2016, 8, 482.

Amadio, M.; Mysiak, J.; Carrera, L.; Koks, E. Improving flood damage assessment models in Italy. Nat. Hazards 2016, 82, 2075-2088

Borah, S.B., Sivasankar, T., Ramya, M.N.S. et al. Flood inundation mapping and monitoring in Kaziranga National Park, Assam using Sentinel-1 SAR data. Environ Monit Assess 190, 520 (2018). https://doi.org/10.1007/s10661-0186893-y

Chaabani, C.; Chini, M.; Abdelfattah, R.; Hostache, R.; Chokmani, K. Flood Mapping in a Complex Environment Using Bistatic TanDEM-X/TerraSAR-X InSAR Coherence. Remote Sens. 2018, 10, 1873.

Chini, M, L. Pulvirenti, and N. Pierdicca, "Analysis and interpretation of the COSMO-SkyMed observations of the 2011 Japan Tsunami," IEEE Geosci. Remote Sens. Lett., vol. 9, no. 3, pp. 467-471, May 2012.

Chini, M.; Pelich, R.; Pulvirenti, L.; Pierdicca, N.; Hostache, R.; Matgen, P. Sentinel-1 InSAR Coherence to Detect Floodwater in Urban Areas: Houston and Hurricane Harvey as A Test Case. Remote Sens. 2019, 11, 107.

Clement, M., Kilsby, C. and Moore, P. (2018), Multi-temporal synthetic aperture radar flood mapping using change detection. J Flood Risk Management, 11: 152-168

Gao, B.C., 1996, NDWI-a normalized difference water index for remote sensing of vegetation liquid water from space. Remote Sensing of Environment, 58, pp. 257-266.

Haralick R. M. and L. G. Shapiro, "Image segmentation techniques," Comput. Vis. Graph. Image Process., vol. 29, no. 1, pp. 100-132, Jan. 1985

Henry, J.-B., Chastanet, P., Fellah, K., Desnos, Y.-L., 2003, July. ENVISAT multipolarised ASAR data for flood mapping. In: Geoscience and Remote Sensing Symposium, 2003. IGARSS'03. Proceedings. 2003 IEEE International. Vol. 2, pp. 1136-1138

Jenks, George F. 1967. "The Data Model Concept in Statistical Mapping", International Yearbook of Cartography 7: 186-190. 
Kornelsen, K.C., Coulibaly, P., 2013. Advances in soil moisture retrieval from synthetic aperture radar and hydrological applications. J. Hydrol. 476 (November), 460-489

Li, J.; Yang, X.; Maffei, C.; Tooth, S.; Yao, G. Applying Independent Component Analysis on Sentinel-2 Imagery to Characterize Geomorphological Responses to an Extreme Flood Event near the Non-Vegetated Río Colorado Terminus, Salar de Uyuni, Bolivia. Remote Sens. 2018, 10, 725

Li Y., S. Martinis, S. Plank, R. Ludwig, An automatic change detection approach for rapid flood mapping in Sentinel-1 SAR data Int. J. Appl. Earth Obs. Geoinf., 73 (2018), pp. 123-135,

Long S., T.E. Fatoyinbo, F. Policelli Flood extent mapping for Namibia using change detection and thresholding with SAR Environ. Res. Lett., 9 (2014), Article 035002

Matgen, P., Hostache, R., Schumann, G.J.-P., Pfister L., Hoffmann L., Savenije,H., 2011. Towards an automated SARbased flood monitoring system: lessons learned from two case studies. Phys. Chem. Earth, Parts A/B/C 36 (January (7-8)), 241-252

Mcfeeters, S.K., 1996, The use of normalized difference water index (NDWI) in the delineation of open water features. International Journal of Remote Sensing, 17, pp.1425-1432.

McFeeters, S.K. Using the Normalized Difference Water Index (NDWI) within a Geographic Information System to Detect Swimming Pools for Mosquito Abatement: A Practical Approach. Remote Sens. 2013, 5, 3544-3561; doi: $10.3390 /$ rs 5073544

McMaster Robert (1997) In Memoriam: George F. Jenks (19161996), Cartography and Geographic Information Systems, 24:1, 56-59, DOI: $10.1559 / 152304097782438764$

Mishra, Abhishek \& Chaudhuri, D. \& Bhattacharya, Chinmoy \& Rao, Ys. (2013). Coherent Change Detection with COSMO SkyMed Data-experimental Results. Defence science journal. Vol. 63. pp. 69-73. 10.14429/dsj.63.3766. 2013

Monti-Guarnieri A. V., M. A. Brovelli, M. Manzoni, M. Mariotti d'Alessandro, M. E. Molinari and D. Oxoli, "Coherent Change Detection for Multipass SAR," in IEEE Transactions on Geoscience and Remote Sensing, vol. 56, no. 11, pp. 68116822, Nov. 2018

Muro, J.; Canty, M.; Conradsen, K.; Hüttich, C.; Nielsen, A.A.; Skriver, H.; Remy, F.; Strauch, A.; Thonfeld, F.; Menz, G. Short-Term Change Detection in Wetlands Using Sentinel-1 Time Series. Remote Sens. 2016, 8, 795.

Notti, D.; Giordan, D.; Caló, F.; Pepe, A.; Zucca, F.; Galve, J.P. Potential and Limitations of Open Satellite Data for Flood Mapping. Remote Sens. 2018, 10, 1673.

Olen, S.; Bookhagen, B. Mapping Damage-Affected Areas after Natural Hazard Events Using Sentinel-1 Coherence Time Series. Remote Sens. 2018, 10, 1272.

Ouled Sghaier, M.; Hammami, I.; Foucher, S.; Lepage, R. Flood Extent Mapping from Time-Series SAR Images Based on Texture Analysis and Data Fusion. Remote Sens. 2018, 10, 237.
Pulvirenti L., M. Chini, N. Pierdicca and G. Boni, "Use of SAR Data for Detecting Floodwater in Urban and Agricultural Areas: The Role of the Interferometric Coherence," in IEEE Transactions on Geoscience and Remote Sensing, vol. 54, no. 3, pp. 1532-1544, March 2016

Rahman, M.S., Di, L. The state of the art of spaceborne remote sensing in flood management. Nat Hazards 85, 1223-1248 (2017)

Refice, A.; Capolongo, D.; Pasquarello, G.; D’Addabbo, A.; Bovenga, F.; Nutricato, R.; Lovergine, P.F.; Pietranera, L. SAR and InSAR for flood monitoring: Examples with COSMOSkyMed data. IEEE J. Sel. Top. Appl. Earth Obs. Remote Sens. 2014, 7, 2711-2722.

Schlaffer, S., Hollaus, M., Wagner, W., Matgen, P., 2012. Flood delineation from synthetic aperture radar data with the help of a priori knowledge from historical acquisitions and digital elevation models in support of near-real-time flood mapping. In: Proceedings of the SPIE, vol. 8538, Earth Resources and Environmental Remote Sensing/GIS Applications III, Edinburgh, 25 October 2012, pp. 853813/1$853813 / 9$.

Slocum, Terry A., and Terry A. Slocum. Thematic Cartography and Geovisualization. Upper Saddle River, NJ: Pearson Prentice Hall, 2009. Print

Sree, Manju \& Srinivasa Rao, Goru \& Brinda, V. \& Veerubhotla, Bhanumurthy. (2006). Advantage of multipolarized SAR data for flood extent delineation - art. no. 64100Z. Proceedings of SPIE - The International Society for Optical Engineering. 6410. 10.1117/12.693947.

Twele Andre, Wenxi Cao, Simon Plank \& Sandro Martinis (2016) Sentinel-1-based flood mapping: a fully automated processing chain, International Journal of Remote Sensing, 37:13, 2990-3004

$\mathrm{Xu}, \mathrm{H}$. Modification of normalised difference water index (NDWI) to enhance open water features in remotely sensed imagery. Int. J. Remote Sens. 2006, 27, 3025-3033. DOI: $10.1080 / 01431160600589179$

Zhang, D.; Zhou, G. Estimation of soil moisture from optical and thermal remote sensing: A review. Sensors 2016, 16, 1308. 